\title{
Diabetic Limb Salvage in the Septic Ankle: Case Studies of Arthrodesis using the Ilizarov Methodology
}

\author{
by Sutpal Singh, DPM. FACFAS ${ }^{1} \underset{\otimes}{ }$, Albert Kim, DPM ${ }^{2}$, Timothy Dailey, DPM ${ }^{3}$, \\ Long Truong, DPM ${ }^{4}$, Maria Mejia, $\mathrm{DPM}^{5}$
}

The Foot and Ankle Online Journal 4 (10): 1

Diabetic patients usually have multiple comorbidities resulting in higher complication rates after ankle fractures. In many cases, the patient, through diabetic complications of peripheral neuropathy, may mistakenly ambulate resulting in dislocation or hardware failure if only internal fixation is utilized. Also, impaired wound healing, infection, non-union, mal-union and development of Charcot foot and ankle arthropathy may ensue. This article will present several cases in which open reduction and internal fixation in diabetic ankle fractures failed which then lead to osteomyelitis. This infection with the presence of diabetic neuropathy results in an increased risk for loss of limb. These cases were ultimately salvaged with septic ankle arthrodesis using the Ilizarov Method.

Key words: Diabetic ankle fracture, osteomyelitis, Limb Salvage, Septic Ankle Arthrodesis, Ilizarov Methodology.

Accepted: September, $2011 \quad$ Published: October, 2011

This is an Open Access article distributed under the terms of the Creative Commons Attribution License. It permits unrestricted use, distribution, and reproduction in any medium, provided the original work is properly cited. OThe Foot and Ankle Online Journal (www.faoj.org), 2011 All rights reserved.

T reating diabetic ankle fractures is a very complex task and many times lead to multiple complications. The majority of diabetic patients have comorbidities such as peripheral vascular disease, osteoporosis with poor bone stock that can lead to poor healing potential and complications. A few of the complications encountered are ulcerations and wound dehiscence.

\footnotetext{
Address correspondence to: Sutpal Singh, DPM. FACFAS, FAPWCA, Chief Ilizarov Surgical Instructor at Doctors Hospital, West Covina, California.

${ }^{1}$ Chief Ilizarov Surgical Instructor at Doctors Hospital, West Covina, California. Private practice in Southern California.

${ }^{2}$ Resident, Doctors of Podiatric Medicine (R3),

3 Resident, Doctors of Podiatric Medicine (R2),

${ }^{4,5}$ Residents, Doctors of Podiatric Medicine (R1).

All residents : Doctors Hospital of West Covina (PM\&S-36).
}

It has been well documented in the literature that diabetic patients with ankle fractures who underwent open reduction and internal fixation developed complications of wound infections, below the knee amputations, Charcot arthropathy, malunions, wound necrosis requiring plastic surgery, and deep sepsis. ${ }^{1,2}$ When complications are encountered, often, salvage is managed by ankle arthrodesis. Our treatment protocol is to fuse the ankle using the Ilizarov Method.

When performing an ankle fusion there are a variety of different open surgical approaches to exposing the ankle for fusion as well as arthroscopic ankle fusions. 


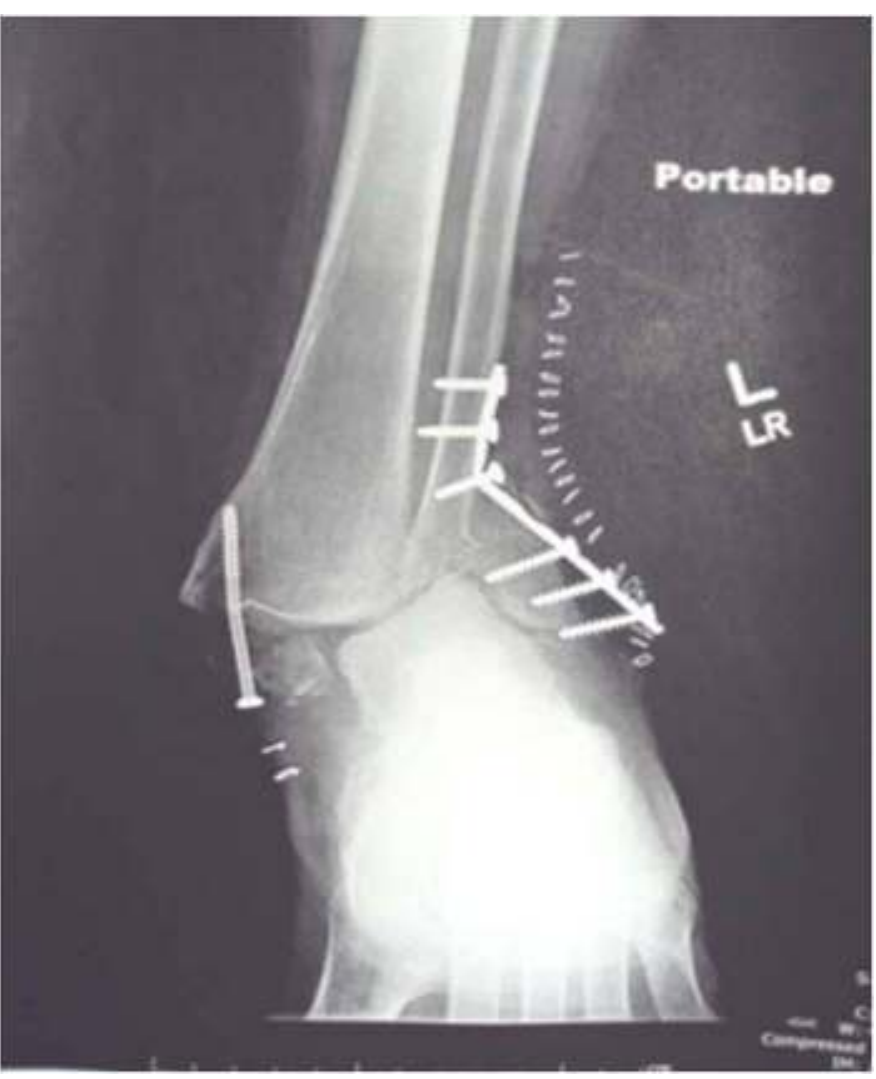

Figure 1 Pre-operative radiograph of failed internal fixation in case \#1.

The open surgical approaches are more commonly used than the arthroscopic option. Of the many different open approaches the more common ones are the medial transmalleolar, lateral transmalleolar, anterior, and posterior approaches. A discussion on the various types of ankle arthodesis will be presented followed by the Ilizarov Method in septic ankle joint arthrodesis.

The medial approach is performed by using an osteotome or oscillating saw to create a transverse medial malleolar osteotomy at the level of the distal tibial articular surface. Next, the medial malleolus is reflected distally on the deltoid hinge, exposing the ankle joint. A power saw is used to resect the tibial plafond perpendicular to long axis of the tibia. Temporary fixation with Steinmann pins can be utilized to maintain the plantigrade position. ${ }^{3}$

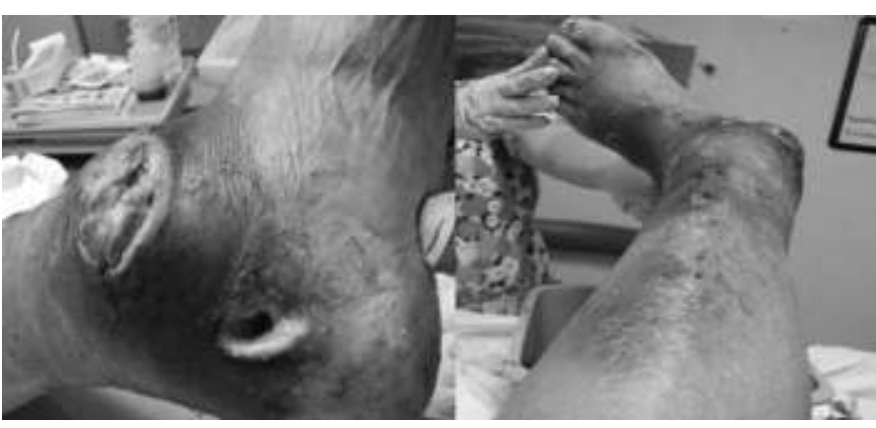

Figure 2 Clinical photograph of the exposed distal tibia and calcaneus in case \# 1.

The medial approach provides better visualization of the tibiotalar articulation ${ }^{3,4,5}$, the surgical exposure obtained is more subcutaneous and gives better access for preparation of the articular surfaces. Neurovascular complications are also decreased by this approach. Finally, the medial approach allows for visualization in placement of a posterior screw which has to be placed blindly when using the lateral approach. ${ }^{3}$

In the lateral approach of ankle arthrodesis, an osteotomy is performed approximately $2 \mathrm{~cm}$ proximal to the level of the ankle joint. In this approach care must be taken not to sever the sural nerve. ${ }^{3,4,5,6}$ The lateral approach is preferred over an anterior approach in cases with moderate to severe deformity. Therefore in cases where a severe deformity of the ankle joint is not present, an anterior approach is indicated.

In regards with the anterior approach, care is taken to avoid any damage to the terminal branches of the superficial peroneal nerve, the intermediate and the medial dorsal cutaneous nerves due to the course of these nerves under the incision site. ${ }^{7}$ The fixation indicated for the anterior approach is composed of at least 2 screws inserted at 30 degrees with respect to the long axis of the tibia. These screws should cross proximal to the fusion site to maximize stability. In certain cases a third screw can be placed to improve sagittal plane stability. ${ }^{7} \quad$ A major advantage of the anterior approach is that the osteotomy of the lateral and medial malleoli is avoided. The other approach which is used but is not discussed in the literature as frequently is the posterior approach. 


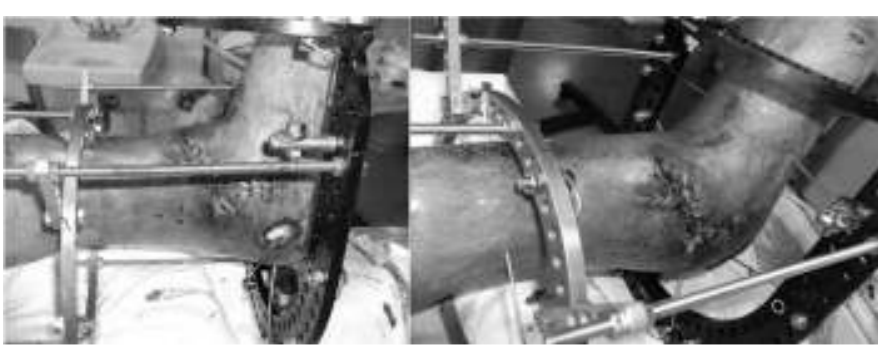

Figure 3 Application of the external fixator, closure of the calcaneal ulcer with a Graft Jacket, and rotational flap to cover the tibial wound at the ankle in case \#1.

For the posterior approach, Hanson et al., concluded that using a posterior approach with a 95 degree blade plate is effective in large patients with a mild to moderate hindfoot deformity.

In addition to the open techniques, an ankle fusion can be performed with arthroscopy. In the arthroscopic technique various abraders, curettes and other arthroscopic instrument are used to remove the cartilage from the joint surfaces using a camera and small portals through the ankle joint. After removing the cartilage the ankle is reduced into proper position and finally fixated internally with two transmalleolar screws. With arthroscopic fusion it has been shown that the average time to fusion is significantly less, whereas this produces a faster recovery period. The shorter time to fusion is likely a result of the minimal soft tissue stripping that is performed during the procedure. ${ }^{4,9}$

Regardless of whether an open or an arthroscopic fusion is performed, the position of the foot for fusion is the same. In the literature the correct position for fusion is valgus of the posterior foot varying from 0 to 5 degrees with an external rotation of 5 to 10 degrees, sloping slightly posterior talus relative to tibia and neutral flexion position. ${ }^{1,2,4,5,6}$ In order to prevent malposition, the foot should be compared to the rest of the leg and the contralateral limb before fusing it.

Once the proper position is found the next concern is fixation. There are various methods of fixation such as compression with an external fixator, internal fixation using plates and screws, intramedullary fixation, and arthroscopic ankle fusion. ${ }^{1,2}$

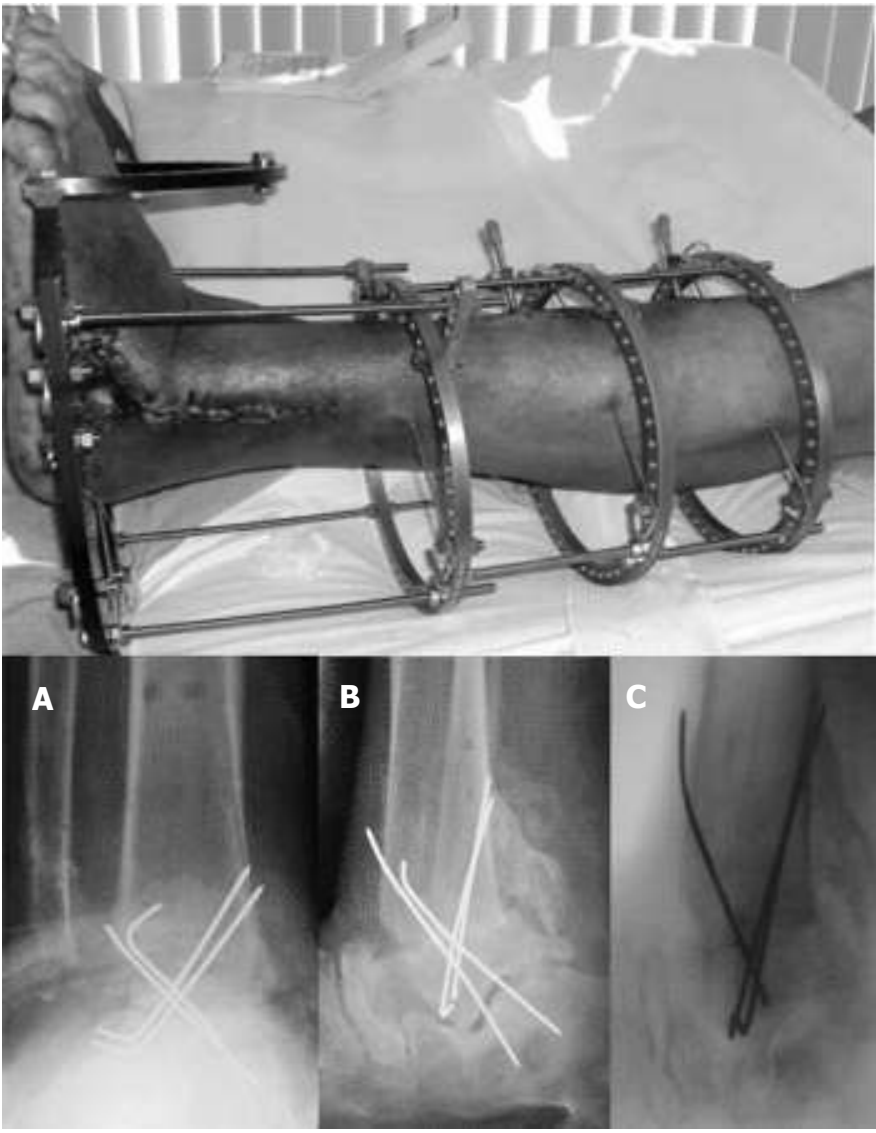

Figure 4 Showing lateral approach in case \#1 with the external fixator. The anteroposterior radiograph (A), Lateral radiograph (B) and lateral radiograph after removal of wires from the subtalar joint (C) in case \# 1. All radiographs showing complete consolidation of the tibial talar joint.

In difficult cases of ankle arthrodesis and limb salvage the preferred fixation methods are Intramedullary (IM) nail and external fixation. 1,4, $^{10,11}$ Obtaining a solid fusion can be challenging in compromised bony interfaces, and standard techniques of tibiotalar fixation such as crossed lag screws are often inadequate. An advantage that the Ilizarov technique has over IM nailing and the other internal fixation options is it can be used in cases of infection. ${ }^{3,4}$ The Ilizarov method also spares the subtalar joint.

Post operative care is comparably the same in almost all the surgical procedures with non weightbearing in a posterior splint followed with a cast for at least 6 weeks, removal of casts depends on healing noted and once healing progresses the patient is placed in a CAM boot. ${ }^{4,5}$ 


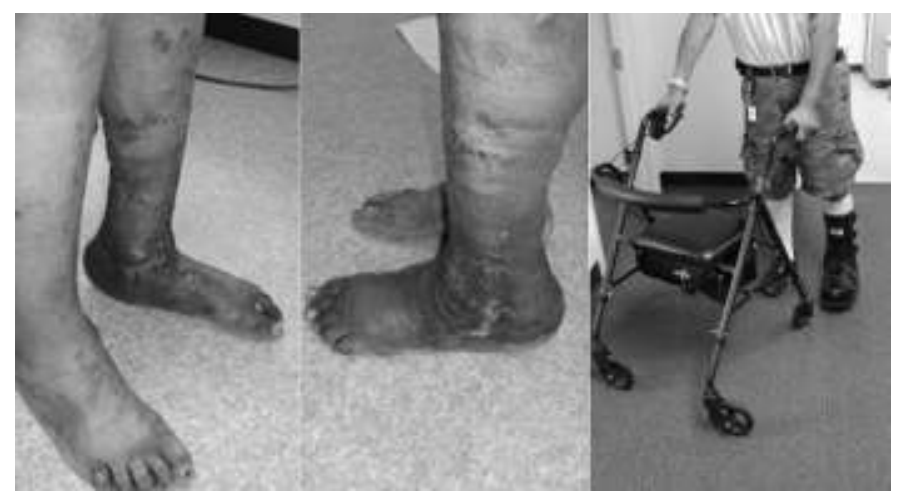

Figure 5 Clinical photographs 4 months after surgery showing limb salvage with all wound healed with solid bone consolidation in case \#1.

For patients that were treated using the Ilizarov Method, early weight bearing is permitted. When comparing open arthrodesis with arthroscopic fusions, the arthroscopic patients were hospitalized for an average of 1.6 (1-4 day range) days whereas the open group was hospitalized for average of 3.4 days (1-6 day range). ${ }^{12}$

As discussed earlier, complications include malposition, neurovascular complications. Nonunions and amputations can also occur as a complication depending on the surgical approach. Initially they can be treated with prolonged periods of immobilization and minimal weightbearing. In addition, an external bone stimulator can be used. ${ }^{11}$ If prolonged immobilization does not help then bone grafting and external fixation are recommended. ${ }^{1,4,5,11,13,14,15}$ In cases in which non unions are painful and they are not able to be resolved with repeated surgical options an amputation is many times the only option. ${ }^{11,13}$ Also superficial infection of the surgical incision or the pin sites in external fixation methods has been reported as occurring in $40 \%$ to $50 \%$ in which local wound care is usually sufficient enough. In cases of deeper infections where osteomyelitis is involved the rate of amputation is as high as $50 \%$ which happens more so in the case of fusions performed in an existing septic process. ${ }^{11} \quad$ This article will present failed open reduction internal fixation (ORIF) in diabetic patients that were salvaged in case of septic ankle fusion using the Ilizarov method in which both medial and lateral incisions were used.

\section{Case Report}

\section{Case \# 1}

The first case is that of a 70 year-old diabetic, neuropathic, cardiomyopathic, liver transplant patient on dialysis. He had a bimalleolar ankle fracture stabilized with internal fixation. (Figs. 1 and 2) $\mathrm{He}$ was referred to our service after undergoing multiple surgeries including wound care and skin graft. He had a large ulcer on the medial and lateral ankle on the left lower extremity. His fibular plate was severely bent and the tibia was exposed on the medial side due to noncompliance and ambulation. An external fixator with several tibial screws and one calcaneal transfixation screw was used to temporarily hold the deformity. This was done prior to being transferred to our service. Cultures revealed Methicillin-resistant Staphylococcus aureus (MRSA) with osteomyelitis at the ankle and the patient was on intravenous (IV) antibiotics. After consulting with infectious disease, internal medicine, cardiology and vascular surgery, the patient was given clearance for limb salvage. The patient had only two choices at the time: (1) below the knee amputation or (2) limb salvage. He chose the latter. The treatment plan included wound care, debridement of the ulcer and removal of the necrotic and infected bone and soft tissue. This was performed 1 week prior to surgery. The patient then underwent a septic ankle arthrodesis using the Ilizarov frame as well as rotational flap to close the ulcer. (Figs. 3-5)

\section{Case \# 2}

This patient is a 380lb, diabetic, neuropathic, cardiomyopathic patient with a malunion. (Fig. 6) $\mathrm{He}$ had an unstable ankle fracture at the fibula with complete rupture of the deltoid ligament. (Fig. 7A and 7B) He was stabilized with open reduction and internal fixation. He ambulated several days after the surgery resulting in malunion and widening of the tibial talar joint. He was seen by our service several months after the initial surgery. He had a large open wound down to the medial tibia with purulent drainage coming from the ankle joint. He presented with osteomyelitis of the ankle. Again, he was cleared for limb salvage. 


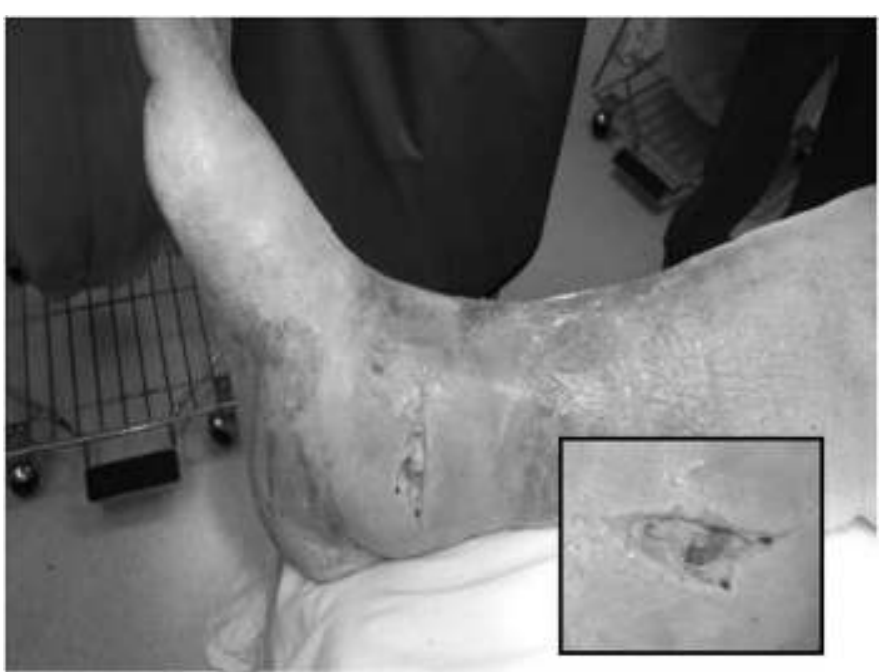

Figure 6 Clinical photographs of open distal tibia with osteomyelitis in case \# 2. (Close-up in inset)

The treatment plan again included wound care, debridement of the ulcer and removal of the necrotic and infected tissue. One week later, he had a septic ankle arthrodesis and subtalar joint arthrodesis using the Ilizarov frame as well as a rotational flap to close the ulcer at the ankle. (Figs. 8A, 8B, 9A, 9B, 10A, 10B, 11 and 12)

\section{Case \# 3}

This patient is a 70 year old diabetic, neuropathic who suffered a severe ankle and foot fracture. She is a chronic tobacco abuser smoking 2 packs per day. She had an ORIF of the right ankle and foot. The patient developed a postoperative infection. She was referred to our service for limb salvage. On initial presentation the patient had a tremendous amount of putrid smelling brown pus coming from the medial ankle. (Fig. 13, 14A and 14B) Culture and sensitivity revealed MRSA. She was on IV Vancomycin. She was taken to the OR and an incision and drainage was performed. The necrotic bone and tissue as well as the hardware at the ankle were removed. The wound was then packed with iodoform and she had daily wound care. One week later, when the infection was controlled, she had a septic ankle arthrodesis using the Ilizarov Method. (Figs. 15, 16A, 16B, 17A, 17B)

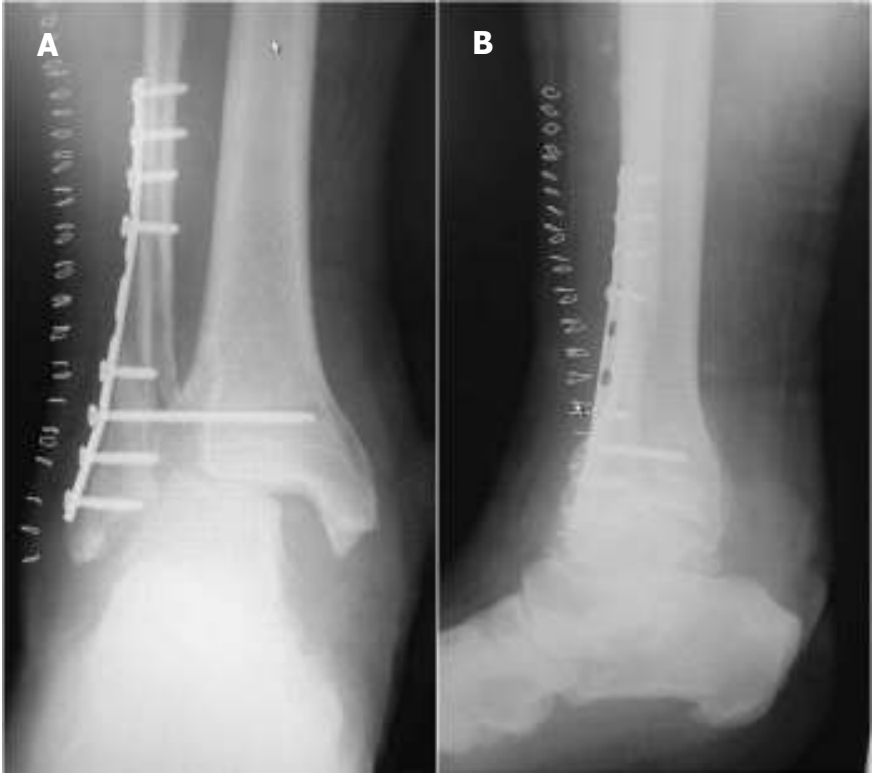

Figure 7A and 7B Anteroposterior (A) and lateral (B) radiographic views of a failed internal fixation resulting in a diabetic septic ankle. (Case \# 2)

\section{Surgical Technique and Result}

Case \# 1: Three tibial rings, each $180 \mathrm{~mm}$ with several smooth $1.8 \mathrm{~mm}$ wires were applied to the proximal tibial segment. Also two half pins were also inserted and attached to the tibial rings. Then a foot plate was applied using the $1.8 \mathrm{~mm}$ wires. Note that the tibial rings and foot plate were not connected at this time. An incision was made on the lateral side. The hardware and the distal fibula were removed. On the medial side, the hardware and distal medial tibia were removed. The ulcer on the medial side was debrided and all necrotic tissue was removed. Then the tibial talar joint was resected until there was good apposition and bleeding. The wound was copiously irrigated with 3 liters of normal saline and bacitracin. The foot plate was manipulated to hold the tibial talar joint in good apposition with the second toe in line with the tibial tuberosity. There was no varus, valgus, dorsiflexion or plantar flexion noted. The tibial talar joint was in neutral position. The foot plate is used to move the foot such that the talus is directly under the tibia and not forward or behind the tibia. Several 0.062 Kirschner wires were inserted to hold the tibial talar joint. (Figs. 3 and 4) 


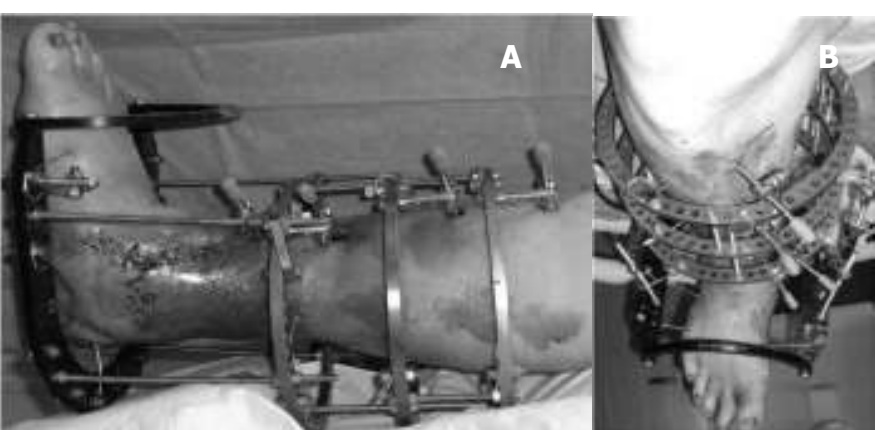

Figure 8A and 8B Clinical photographs of septic ankle arthrodesis using the Ilizarov frame. Medial view: Note closure of the ankle using a rotational flap (A) and anterior view. This is the second day after surgery for case \#2.

Rods were then applied to the foot plate and tibial rings. Compression was applied in an axial direction. There was good alignment and good compression. Another incision was made above the ulcer and a full thickness rotational flap was performed to close the ulcer where the tibia was exposed. There were also two other ulcers noted which were created by the prior transfixation screw through the calcaneus. These ulcers were debrided to good bleeding tissue and then covered with Graft Jacket and sutured with 3-0 Prolene ${ }^{\mathrm{TM}}$. The rest of the surgical sites were closed using 3-0 Vicryl ${ }^{\mathrm{TM}}$ for deep tissue and 3-0 Prolene ${ }^{\mathrm{TM}}$ for the skin as well as skin staples.

The external fixation was left on for three months until consolidation was seen on radiograph. Then the external fixation was removed and the wires going into the subtalar joint was removed. A CROW boot was then dispensed to the patient to protect the limb. The patient then began ambulating with a walker. At six month and one year follow-up, the patient is still ambulating and without any recurrence. (Fig. 5)

Case \# 2: Three $200 \mathrm{~mm}$ tibial rings were applied to the patient proximal to the open wound on the lower leg. Then $1.8 \mathrm{~mm}$ smooth wires were inserted and tensioned appropriately. Then 4 tibial half pins were inserted into the tibia and attached to the tibial rings.

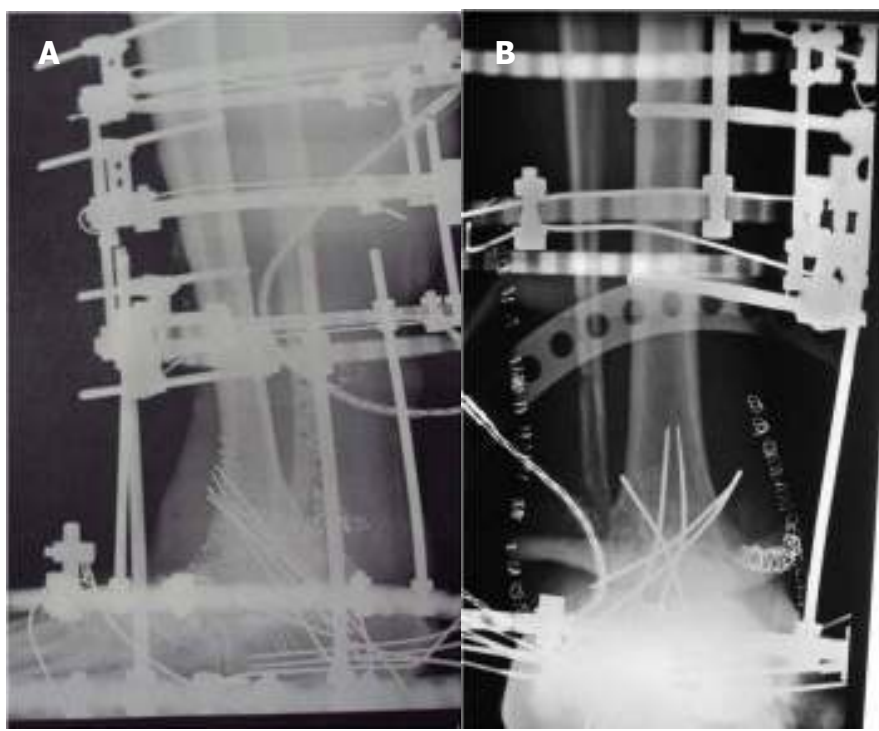

Figure 9A and 9B Lateral (A) and anteroposterior (B) radiographic views 2 days after surgery showing ankle and subtalar joint arthrodesis compressed with an Ilizarov circular external fixator in case \# 2.

Then a foot plate was applied to the foot with wires and tensioned appropriately. The tibial rings and the foot plate were not connected. Then an incision was made on the medial and lateral ankle. All the necrotic bone, tissue and the hardware were then removed. The tibia and the talus were then resected to good bleeding tissue and good apposition. The lateral incision was also extended to the subtalar joint and the subtalar joint was then denuded of cartilage. The large ulcer on the medial side was debrided and all necrotic tissue was removed. There was an even larger opening on the medial side after the debridement. The surgical site was irrigated with 3 liters of normal saline with bacitracin. The foot plate with the foot was then manipulated in a manner in which the tibia and second toe was in line. The tibial talar joint was in neutral position without varus or valgus. There was no varus, valgus, dorsiflexion or plantar flexion noted. The tibial talar joint was in neutral position. The foot plate is used to move the foot such that the talus is directly under the tibia and not forward or behind the tibia. Then several 0.062 Kirschner wires were inserted from the calcaneus, through the talus and then into the tibia. 


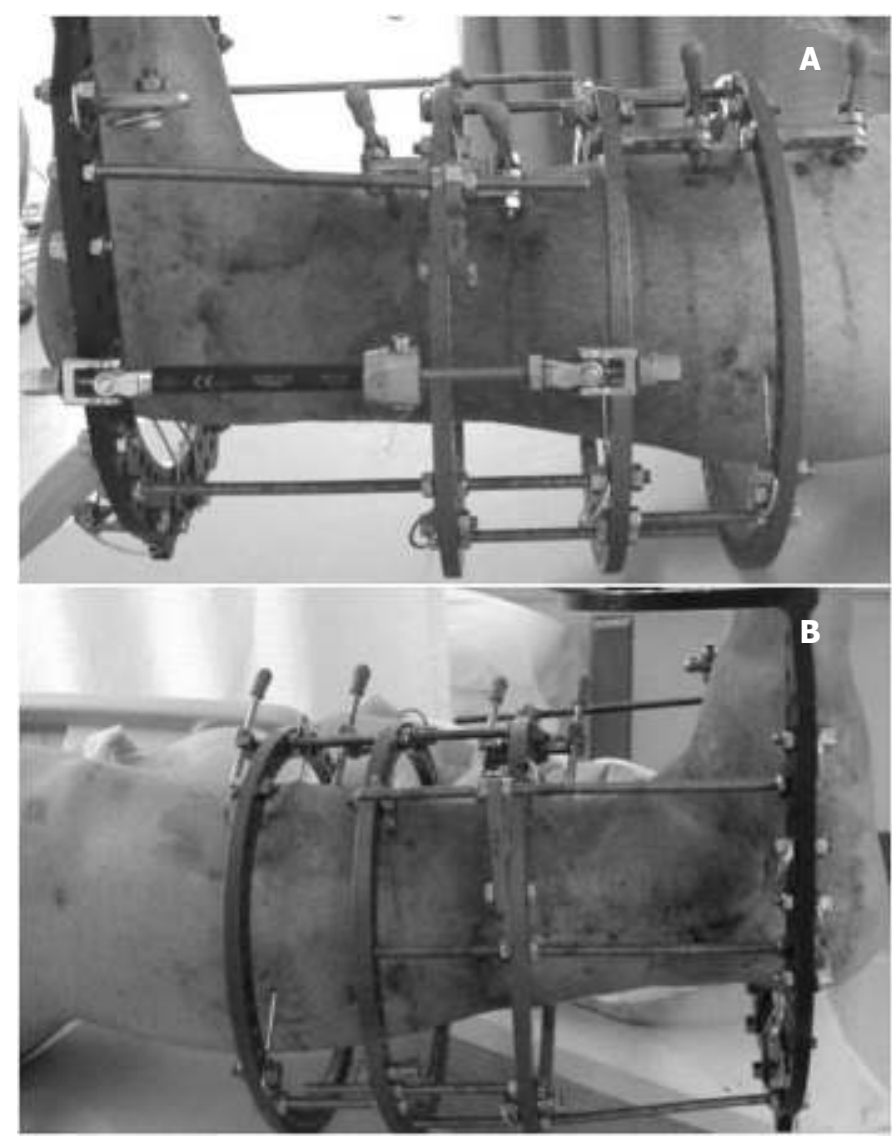

Figure 10A and 10B Medial (A) and lateral (B) views 3 months after tibial-talar-calcaneal fusion with the Ilizarov frame. The skin on the medial side has completely healed with the rotational flap in case \# 2 .

Rods were then used to connect the foot plate to the tibial rings and this was then compressed to fuse the tibial-talar-calcaneal joint. Attention was then directed to the medial large ulcer. Another incision was made at the ulcer and a rotational flap was performed so as to close the ulcer. The surgical site was closed with 3-0 vicryl for the deep tissue, and 3-0 Prolene ${ }^{\mathrm{TM}}$ and skin staples for the skin.

The external fixator was left in place for three months until good consolidation was noted. The $\mathrm{K}$ wires were removed. Because he was morbidly obese, internal splinting with percutaneous 6.5 bolt screws from Synthes were inserted from plantar calcaneus to the tibia. He was also given custom AFO. (Figs. 8A,8B, 9A, 9B, 10A, 10B, 11 and 12) At six month and one year follow-up, the patient is still ambulating and without any recurrence.

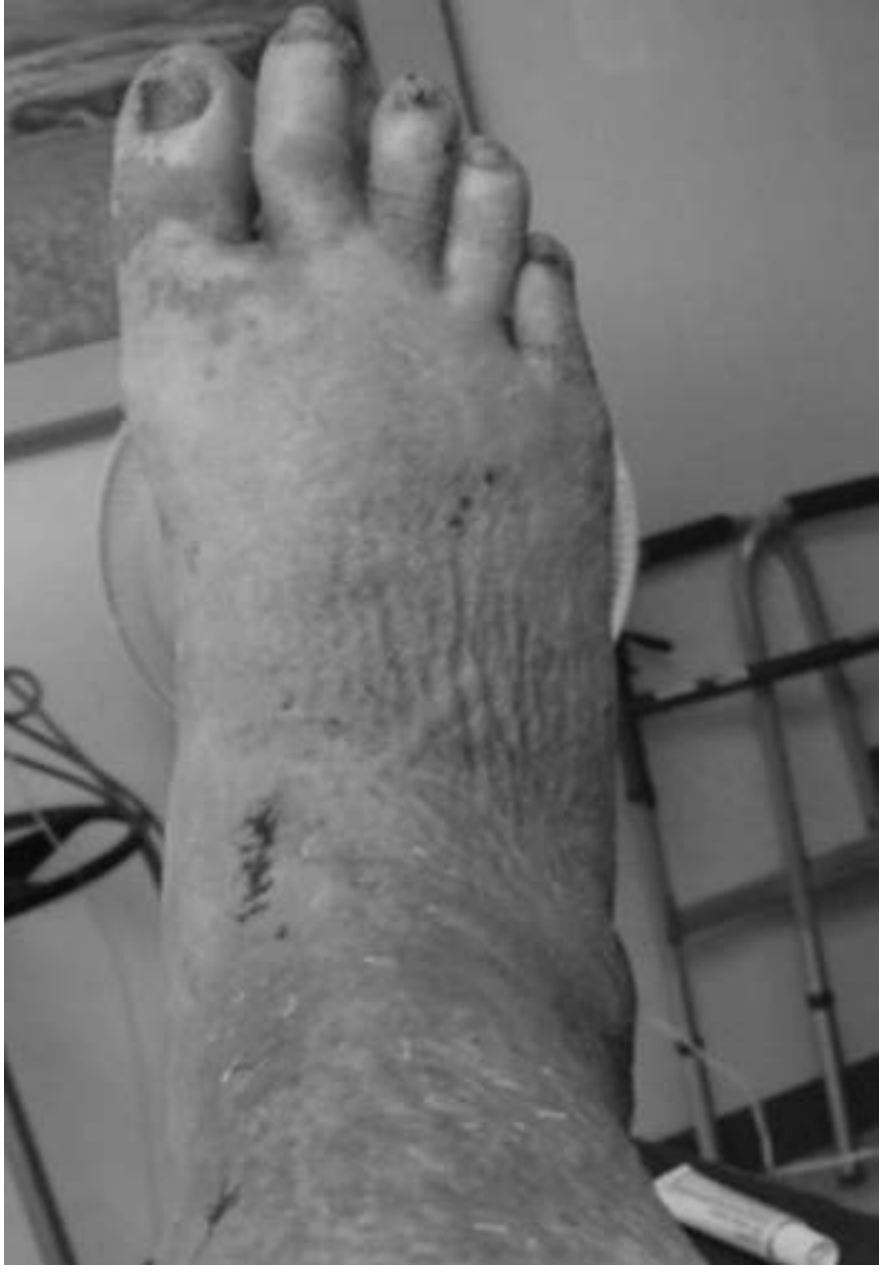

Figure 11 The ulcer has completely healed and the Ilizarov external fixator has been removed in case \#2. The foot is very stable and completely fused at the tibial-talar-calcaneal joint.

Case \# 3: The patient had severe abscess at the medial left ankle with the pus tracking laterally up the leg. (Fig. 13) An incision and drainage was performed on the medial and lateral ankle. The infected tissue, bone and hardware were all removed as well at the distal fibula. The surgical site was irrigated copiously with three liters of normal saline and bacitracin using a pulse lavage system. The surgical site was loosely approximated with 3-0 Prolene $^{\mathrm{TM}}$ and skin staples. She then had wound care every day including the use of Betadine ${ }^{\circledR}$ soaked iodoform as well as irrigation with one liter of normal saline and bacitracin for 5 days. Once the infection was controlled, she was then taken back to the OR for a septic ankle arthrodesis. 


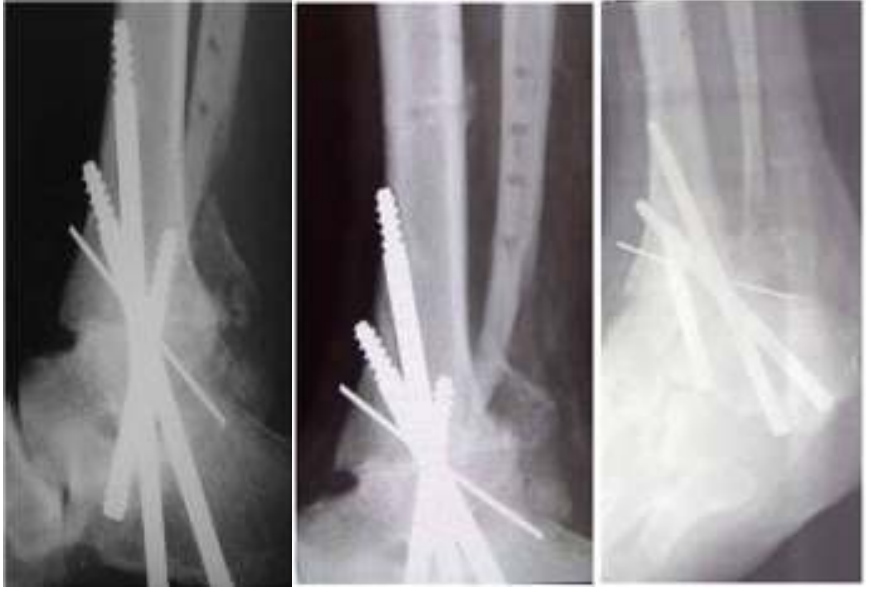

Figures 12 Post operative radiographs showing complete arthrodesis of the tibial-talar-calcaneal joint and stabilization using percutaneous $6.5 \mathrm{~mm}$ fusion Synthes bolts after the Ilizarov frame was removed in case \#2.

The patient was taken back to the OR and three 180 $\mathrm{mm}$ tibial rings were applied to the left lower leg proximal to the infected area. (Fig. 15) The wires were tensioned appropriately and then 2 half pins were applied. Then a foot plate was applied and tensioned appropriately. The tibial talar joint was then resected and then placed in a neutral position without any varus or valgus. There was no varus, valgus, dorsiflexion or plantar flexion noted at the tibial talar joint. Also note that the foot plate is used to move the foot such that the talus is directly under the tibia and not forward or behind the tibia. Her tibial talar joint was in neutral position. This was then stabilized with several $0.062 \mathrm{~K}$ wires. The foot plate with the foot was then connected to the tibial rings with several rods. These were then tightened to compress the tibial talar joint. She did have a valgus tilt of the subtalar joint with the heel being laterally located. (Figs. 16A and 16B) Because of the complexity of the deformity, it was decided to perform a medial calcaneal slide osteotomy at a different time until there was complete consolidation of the tibial talar joint. Antibiotic beads of $1 \mathrm{gm}$ of Vancomycin were made and inserted into the lower leg ankle area.

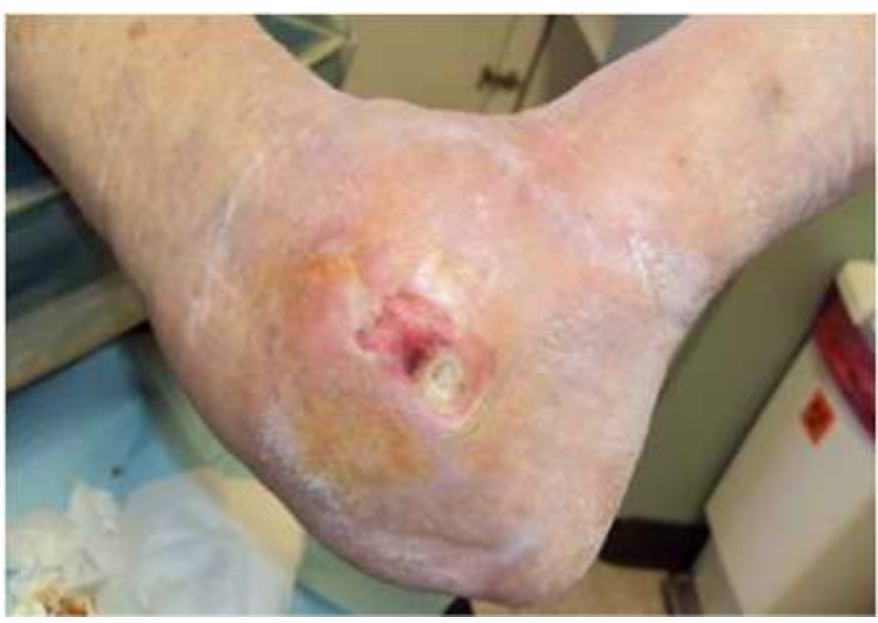

Figure 13 Clinical photograph showing the diabetic open septic ankle joint. The toes are to the upper right and the knee is to the upper left. (Case \#3)

The external fixator was left in place for four months until good consolidation was noted. The $\mathrm{K}$ wires were removed. At this time, because of the severe deformity and possibility of recurrence and BKA, internal splinting with a 10-hole $3.5 \mathrm{~mm}$ metaphyseal plate and screws spanning the tibial-talar-calcaneal complex was performed. Also, a medial calcaneal slide osteotomy was also performed to have a more rectus foot and in better alignment of the leg and hindfoot. This was performed by making an incision on the lateral calcaneal area. The incision was deepened to the subcutaneous tissue and then to bone. A sagittal saw was used to perform the osteotomy and the calcaneus was translated medially approximately $2 \mathrm{~cm}$ and stabilized with several crossing $\mathrm{K}$ wires. (Figs. 17A and 17B)

Two months later, the wires in the calcaneus were removed and a custom Arizona brace was dispensed and she was able to ambulate with a walker.

After six months, she developed an ulcer on the plantar right foot. She had a Charcot foot prior to the severe infection on the left foot. This ulcerated after six months but with proper wound care, this ulcer completely healed. She was also dispensed another Arizona brace for the right lower extremity. At two year follow-up, she is doing well and has both of her legs and feet. (Figs. 18,19) 

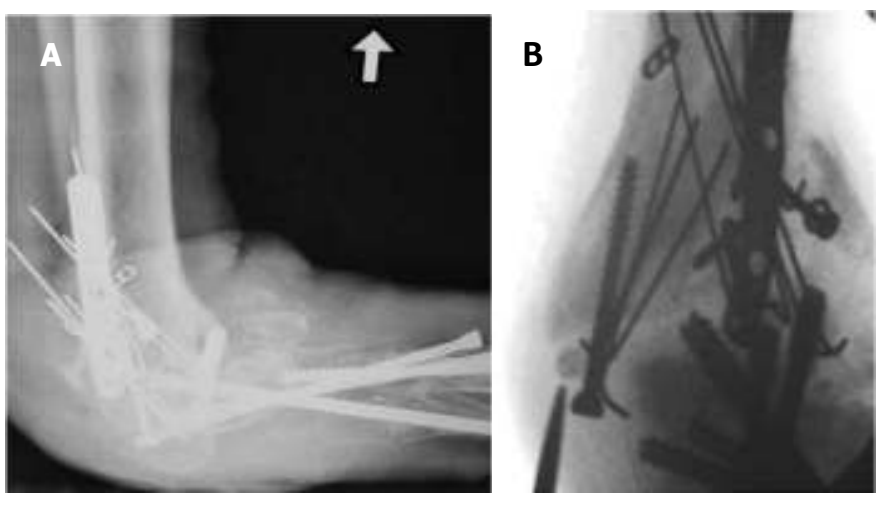

Figure 14A and 14B Severe foot and ankle deformity with sepsis at the tibial talar joint and failed hardware. (A) Note the probe in the medial ankle. (B) Putrid smelling brown pus was noted coming from the medial ankle and tracking across the ankle to the lateral mid leg area in case \#3.

\section{Discussion}

Multiple studies have noted that open reduction internal fixation in acute diabetic ankles fracture can be devastating. ${ }^{16,17,18,19,20}$ Patients with complications associated with diabetes are at an increased risk for higher rates of in hospital mortality, in hospital post operative complications, length of stay and nonroutine discharges. ${ }^{19}$ Previous studies has shown mortality rate as high as $8.5 \%$ and deep infection of $17 \%$ associated with complications of diabetic ankle fracture. $^{18}$ Even after anatomical reduction with stable internal fixation, the diabetic neuropathic patients may experience complications such as breaking or bending the fibular plate, malunion, nonunion, and charcot arthropathy. After repeat ORIF of the ankle with stacked one-third tubular plates and several syndesmotic screws, failure can occur. It is noted in previous studies that diabetic neuropathic patients are 5 times more likely to need revision surgery when comparing to patient with uncomplicated diabetes. ${ }^{20}$ Salvage by tibiotalocalcaneal fusion with intramedullary rod in this population group also failed due to non compliance. This can ultimately resulted in a below the knee amputation. ${ }^{2,21}$ In revisions surgery, the fusion rate is noted to be lower than in primary arthrodesis. $^{22}$

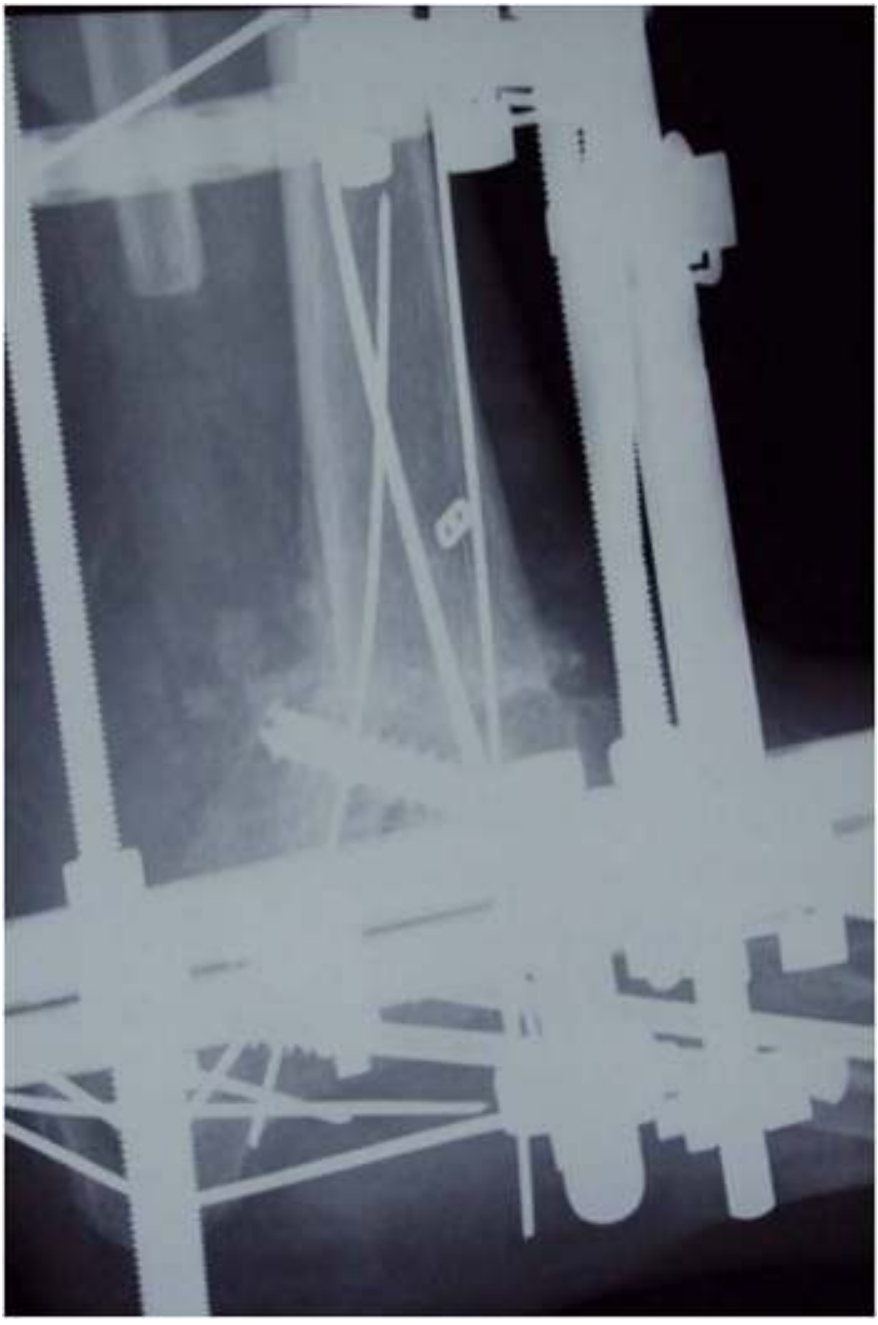

Figure 15 Lateral radiographic view of the septic ankle arthrodesis using the Ilizarov frame several weeks after surgery. There is good alignment of the tibial talar complex. There is placement of antibiotic beads in the ankle/lower leg area. (Case \#3)

Thus, in our case reports, our protocol is to perform ORIF and then to stabilize the lower extremity with an Ilizarov frame. If the patient has peripheral vascular disease, the Ilizarov frame was applied with very minimal to no internal fixation. If the patient is severely medically compromised, the surgery was performed under IV sedation using a popliteal block, common peroneal block at the neck of the fibula and saphenous nerve block at the level of the tibial tuberosity. 


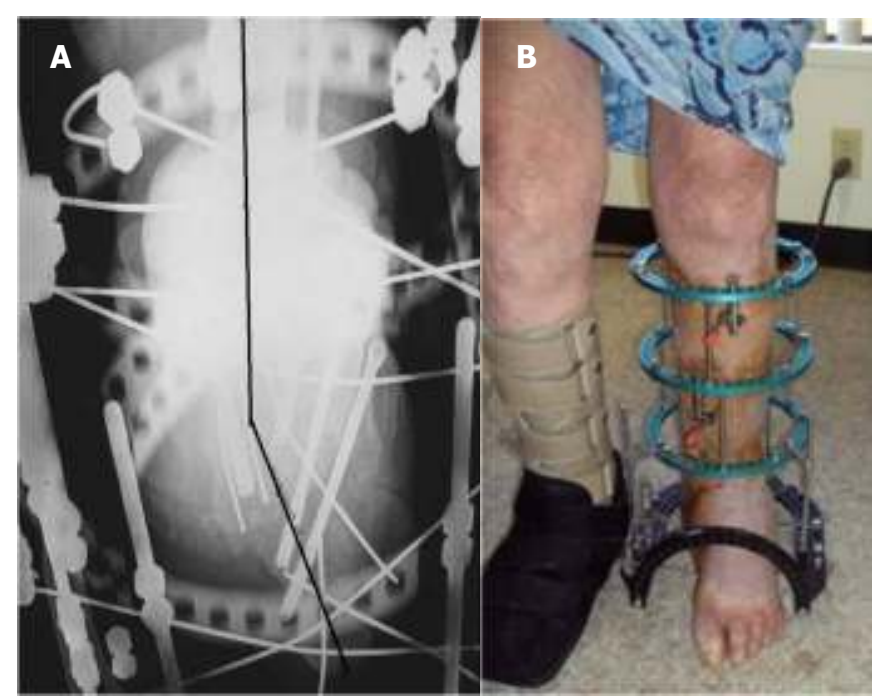

Figure 16A and 16B There is a valgus rotation of the calcaneus relative to the long axis of the tibia. (A) The forefoot was in neutral position without any varus or valgus. (B) All incisions have healed. (Case \# 3)

Several surgical techniques are currently accepted for performing primary ankle arthrodesis. These techniques include compression with an external fixator, internal fixation using plates and screws, intramedullary fixation, and arthroscopic ankle fusion. $^{1,22}$

The Ilizarov technique offers several advantages that "traditional" fusion does not offer in patients with complex ankle pathology such as infection, limblength discrepancy, mal-union, Charcot joints, talar osteonecrosis, and talar absence. ${ }^{22}$ Internal fixation and arthroscopic techniques are not suitable methods for infection, bone loss, severe deformities, or failed procedures. ${ }^{1}$ Post-operatively, the Ilizarov method allows for adjustments in mechanical control throughout the treatment period that is otherwise impossible with nails, screws, or plates. ${ }^{1,22}$

Potential limitations that can be associated with this technique include pin track problem, the cumbersome frame, and complexity associated with application of the frame. ${ }^{22}$

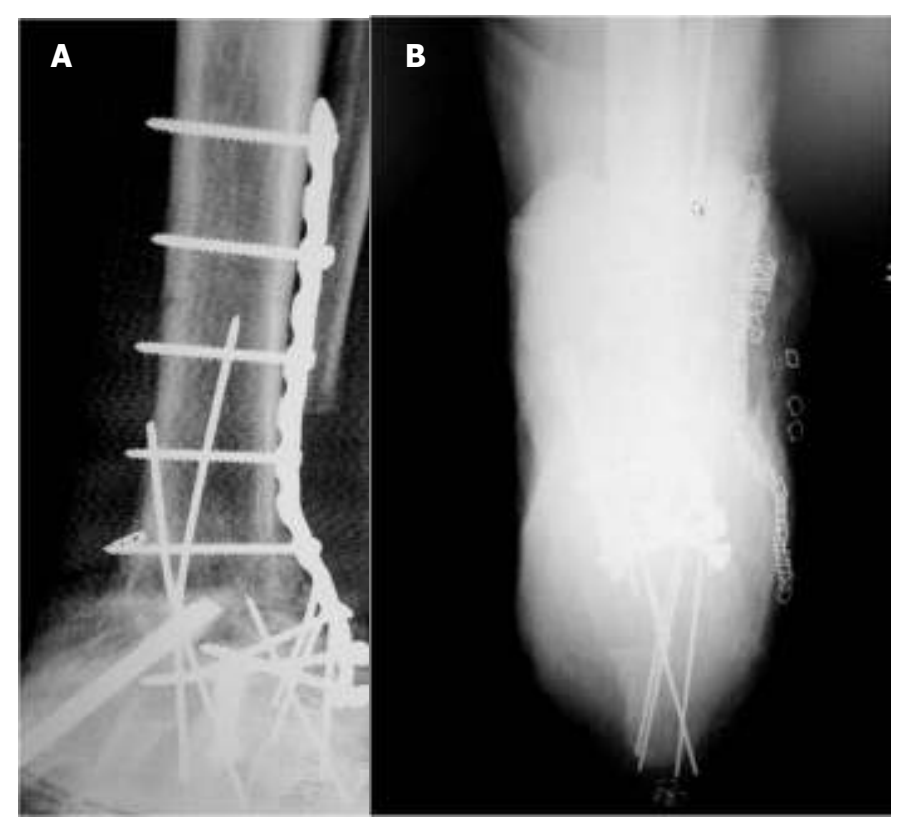

Figure 17A and 17B After removal of the external fixator, insertion of internal splinting with Synthes metaphyseal plate, and calcaneal osteotomy with medial translation. This shows good alignment of the lower extremity. The tibial talar joint is completely fused. (A) Lower leg, ankle and hind foot are in good alignment after the medial calcaneal slide osteotomy. (B) (Case \#3)

Pin track infections do occur but usually are managed locally with pin site care and oral antibiotics. However, the advantages outweigh these downfalls. These advantages prove even more invaluable when application is planned for revisions and complex situations. ${ }^{1}$

The Ilizarov technique provides stable fixation and allows application of primary and continuous forces along any axis and direction. ${ }^{1,22}$ The dynamic axial fixation maintains bone contact without additional bone grafting and allows excellent bending, shearing, and torsional stability that allows early weightbearing. ${ }^{1,22}$ Most patients are bearing partial weight immediately, therefore earlier compression is noted across the surgical site, enhancing fusion rate. ${ }^{22}$ Additionally, due to early ambulation, there is noted improvement in proprioception and reduction in complications such as deep vein thrombosis and deconditioning. ${ }^{22}$ 


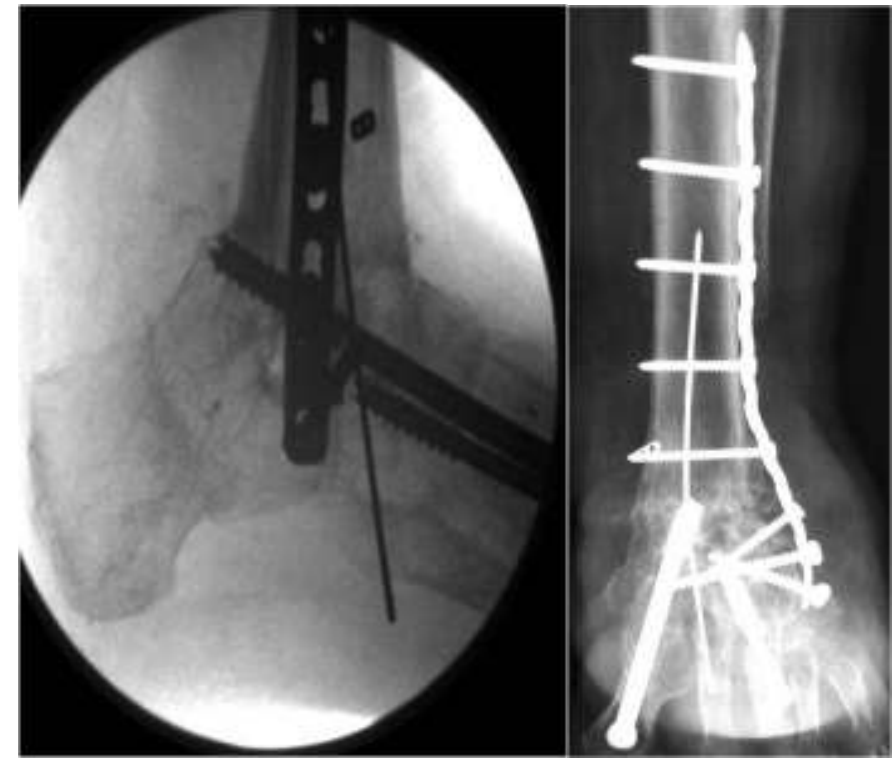

Figures 18 Two years after surgery, the radiographs show good alignment and complete arthrodesis of the tibial-talar-calcaneal bones. The wires in the posterior calcaneus have all been removed in case \#3.

The Ilizarov technique also enables correction in a single plane or in multiple planes. ${ }^{1,2,22}$ A well aligned fusion ensures a near normal gait. It is recommended that fusion be position with valgus of the posterior foot varying from 0 to 5 degrees with an external rotation of 5 to 10 degrees, sloping slightly posterior and neutral flexion position. ${ }^{1,2}$

Ankle arthrodesis can be divided by approach as anterior, transmalleolar, or posterior or by method of fixation as external or internal. ${ }^{1}$ However, when planning the proper procedure for the high risk diabetic patient, many techniques become less appropriate with frequent complications and difficult to achieve fusion site. In our case reports in this article, we performed septic ankle arthrodesis using the Ilizarov Method for limb salvage. All patients were told that a BKA was eminent. We were able to salvage the limb by the Ilizarov Methodology. It has been noted by Gabriel Ilizarov that osteomyelitis burns in the fury of osteogenesis. Osteogenesis occurs by compression and immobilization of bone using the Ilizarov Methodology. In the presence of infection of the tibiotalar joint, arthrodesis is a reasonable treatment option and in some cases may be the way to prevent amputation at a more proximal level. $^{2}$

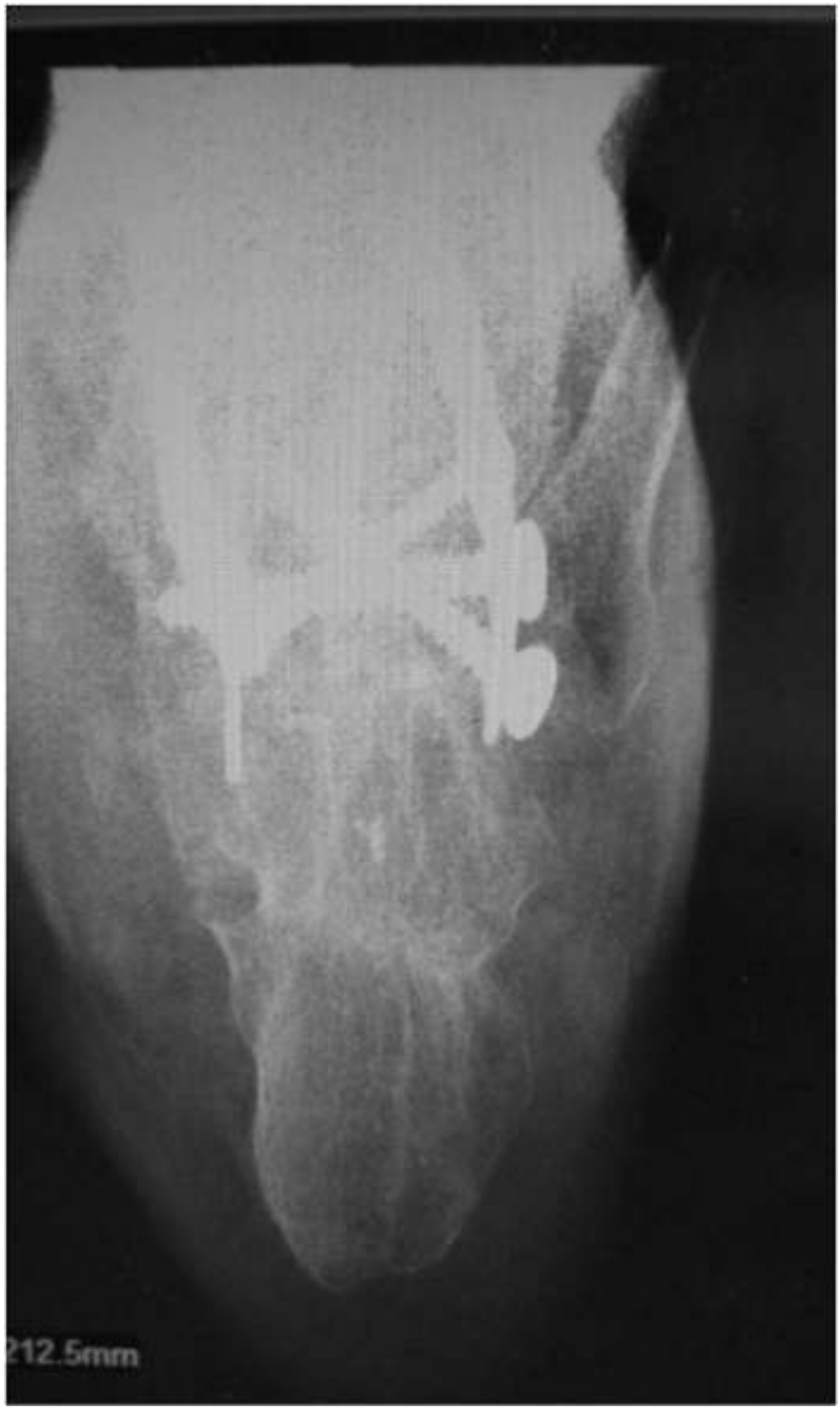

Figure 19 Complete healing of the calcaneal osteotomy in anatomic good position after removal of the internal fixation wires in case \#3.

\section{Conclusion}

The previous cases of diabetic ankle fractures which were fixed with open reduction and internal fixation went on to septic ankle joints. Septic ankle joint is a difficult condition to treat with two viable options limb salvage ankle arthrodesis or below knee amputation. Patients must be aware that ankle arthrodesis may still end up in a BKA. Many different ankle arthrodesis surgical techniques exist with the salvage option. 
Each surgeon has his or her preference as to their procedure of choice with each having their advantages and disadvantages. The author's systematic approach to diabetic ankle fractures is to cast if they are non-displaced, and ORIF with an Ilizarov frame if ankle fracture is displaced. If they go on to a septic ankle joint then the area is debrided and internal hardware is removed and an Ilizarov method is used for ankle arthrodesis. In the authors experience the biggest complication with the Ilizarov frame is pin tract irritations and or infections but these are easily treated by removing the pin and placing a new one. The Ilizarov method is a good option in providing adequate compression and in allowing the patient to bear weight. It is important to follow these patients frequently to make sure the arthrodesis site is healing well and free of infections to prevent a BKA.

\section{References}

1. Salem KH, Kinzl L, Schmelz A. Ankle arthrodesis using Ilizarov ring fixators: A review of 22 cases. Foot \& Ankle International 2006 27:764-70.

2. Klouche S, El-Masri F, Graff W, Mamoudy P. Arthrodesis with internal fixation of the infected ankle. J Foot \& Ankle Surgery 2011 50: 25-30.

3. Schuberth J, Cheung C, Rush S, Blitz N, Roling B. The medial malleolar approach for arthrodesis of the ankle: A report of 13 cases. J of Foot \& Ankle Surgery 2005 44:125-132.

4. Easley M. Operative Techniques in Foot and Ankle Surgery.

Philadelphia: Lippincott Williams \& Wilkins 2011.

5. Coughlin M, Mann R, Saltzman C: Surgery of the Foot and Ankle. Philadelphia. Mosby 2007.

6. Grass R, Rammelt S, Biewener A, Zwipp H: Arthrodesis of the ankle Joint" Clinics Podiatric Medicine Surgery 2004 21:161178.

7. Karl-Heinz K, Hans-Jörg T, Fusszentrum W. Ankle arthrodesis with an anterior approach. Techniques Foot Ankle Surgery 2007 6: 243-248.

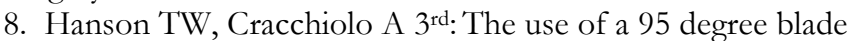
plate and a posterior approach to achieve tibiotalocalcaneal arthrodesis. Foot Ankle International 2002 23:704-710.
9. Glick J, Morgan C, Myerson M, Sampson T, Mann J. Ankle arthrodesis an arthroscopic method: Long-term follow-up of 34 Cases. Arthroscopy 1996 12: 428-434.

10. Fragomen AT, Fragomen AT, Meyers KN, Davis N, Shu H, Wright T, Rozbruch SR. A biomechanical comparison of micromotion after ankle fusion using 2 fixation techniques: Intramedullary arthrodesis nail or Ilizarov external fixator. Foot \& Ankle International 2008 29: 334-341.

11. Raikin S, Venkat R. An approach to the failed ankle arthrodesis. Foot Ankle Clinics 2008 13:401-416.

12. O'Brien T, Hart T, Shereff M, Stone J, Johnson J. Open versus arthroscopic ankle arthrodesis A comparative study. Foot Ankle International 1999 20: 368-373.

13. Hagen RJ. Ankle arthrodesis: problems and pitfalls. Clinical Orthopaedics and Related Research. 1986 202: 152-162.

14. Katsenis D, Bhave A, Paley D. Treatment of malunion and nonunion at the site of an ankle fusion with the Ilizarov apparatus. JBJS 2005 87A: 302-309.

15. Morgan CD, Henke JA, Bailey RW, Kaufer H. Long-term results of tibiotalar arthrodesis. JBJS 1985 67A: 546-550.

16. Costigan W, Thordarson D, Debnath U. Operative management of ankle fractures in patients with diabetes mellitus. Foot \& Ankle International 2007 28: 32-37.

17. Jones KB, Maiers-Yelden KA, Marsh JL, Zimmerman MB, Estin M, Saltzman CL. Ankle fractures in patients with diabetes mellitus. JBJS 2005 87B: 489-495.

18. McCormack R.G., Leith J.M.: Ankle fractures in diabetics: Complications of Surgical Management. JBJS1998 80B: 689692.

19. Wukich D, Joseph A, Ryan M, Ramirez C, Irrgang JJ. Outcomes of ankle fractures in patients with uncomplicated versus complicated diabetes. Foot \& Ankle International 2011 32:120-30.

20. Kline AJ, Gruen GS, Pape HC, Tarkin IS, Irrgang JJ, Wukich DK. Early complications following the operative treatment of pilon fractures with and without diabetes. Foot \& Ankle International 2009 30:1042-1047.

21. Thordarson, D: Ankle fractures in diabetics. Techniques in Foot and Ankle Surgery. 2004 3: 192-197.

22. Eylon S, Porat S, Bor N, Leibner E. Outcome of Ilizarov ankle arthrodesis. Foot \& Ankle International. 2007 28: 873-879. 